\title{
An approach for the identification of microRNA with an application to Anopheles gambiae
}

\author{
Raghunath Chatterjee and Keya Chaudhuri ${ }^{\bowtie}$ \\ Human Genetics \& Genomics Group, Indian Institute of Chemical Biology, Jadavpur, Kolkata, India; \\ ®e-mail: kchaudhuri@iicb.res.in
}

Received: 17 February, 2006; revised: 23 April, 2006; accepted: 08 May, 2006 available on-line: 13 June, 2006

\begin{abstract}
MicroRNAs (miRNAs) are an abundant class of 20-27 nt long noncoding RNAs, involved in posttranscriptional regulation of genes in eukaryotes. These miRNAs are usually highly conserved between the genomes of related organisms and their pre-miRNA transcript, about 60-120 nt long, forms extended stem-loop structure. Keeping these facts in mind miRsearch is developed which relies on searching the homologues of all known miRNAs of one organism in the genome of a related organism allowing few mismatches depending on the phylogenetic distance between them, followed by assessing for the capability of formation of stem-loop structure. The precursor sequences so obtained were then screened through the RNA folding program MFOLD selecting the cut-off values on the basis of known Drosophila melanogaster pre-miRNAs. With this approach, about 91 probable candidate miRNAs along with pre-miRNAs were identified in Anopheles gambiae using known D. melanogaster miRNAs. Out of these, 41 probable miRNAs have $100 \%$ similarity with already known $D$. melanogaster miRNAs and others were found to be at least $85 \%$ similar to the miRNAs of various other organisms.
\end{abstract}

Keywords: microRNA, Anopheles gambiae, RNA folding

\section{INTRODUCTION}

MicroRNAs (miRNAs) are an abundant class of small 20 to 27 nucleotide (nt) noncoding RNAs found in diverse organisms, both plants (Bartel \& Bartel, 2003) and animals (Lim et al., 2003a). Many of these are known to control the expression of other genes at the post transcriptional level (LagosQuintana et al., 2001; Lau et al., 2001; Lee \& Ambros, 2001; Moss \& Poethig, 2002; Bartel, 2004). The founding members of this class of noncoding RNAs are the lin-4 and let-7 gene products of Caenorhabditis elegans (Lee et al., 1993; Reinhart et al., 2000). Both lin-4 and let-7 RNAs act as repressor of their respective target genes lin-14, lin-28 and lin-41 (Lee et al., 1993; Moss et al., 1997; Slack et al., 2000). In all these cases repression was mediated by the presence of complementary miRNA sequences in the $3^{\prime}$ untranslated regions (UTRs) of the target mRNAs (Slack et al., 2000; Lewis et al., 2003).

The unique characteristics of miRNA are first, all miRNAs are present in noncoding regions of the genome; second, when genomic sequences surrounding the identified 22 nt RNAs were examined, computer analysis predicted miRNA precursors capable of forming stem-loop structure, a single miRNA molecule ultimately accumulates from one arm of each miRNA hairpin precursor molecule; third, miRNA sequences are nearly always conserved in related organisms.

To identify novel miRNAs, several approaches have been used involving biochemical approach based on purification of RNAs after size fractionation (Lau et al., 2001) or bioinformatics approach centering on the conservation of intergenic regions of DNA between two clearly related Caenorhabditis species (Lim et al., 2003b) or between Drosophila melanogaster and Drosophila pseudoobscura species (Lai et al., 2003). Both miRscan and MiRseeker extracted conserved intergenic regions between two closely related species. MiRseeker subjects conserved intronic and intergenic sequences to an RNA folding and evaluation procedure to identify evolutionarily constrained hairpin structures with features charac- 
teristic of known miRNAs (Lai et al., 2003). On the other hand, miRscan evaluates conserved stem-loops as miRNA precursors by passing a 21-nt window along each conserved stem-loop, assigning a loglikelihood score to each window that measures how well its attributes resemble those of the first experimentally verified C. elegans miRNAs with $C$. briggsae homologs (Lim et al., 2003b).

For the detection of novel miRNAs in specific animals and plants, comparative genomics was used in several reports (Lim et al., 2003a; 2003b; Lai et al., 2003; Bonnet et al., 2004; Jones-Rhoades \& Bartel, 2004; Ohler et al., 2004; Wang et al., 2004) and for the detection of orthologs and paralogs of known miRNAs, homology searching was also used (Pasquinelli et al., 2000; Lagos-Quintana et al., 2001; Lau et al., 2001; Lee \& Ambros, 2001; Weber, 2005). Using the current sequence alignment tools like blast (Altschul et al., 1990), short sequences (mature miRNAs are of about $22 \mathrm{nt}$ ) as a query sequence will produce large number of irrelevant hits. Pre-miRNA sequences are also used for homologue searching. But due to the non-conservation of the other parts compared with miRNA and miRNA* (the fragments on the opposite arm of the hairpin) (Lau et al., 2001), it is expected that, the homology searching based on pre-miRNA sequences may produce many false positives. So, the more sensitive approach will be to consider both sequence and structure conservation. Using this strategy, ERPIN used pro-files to capture both sequence and structure information of animal miRNA precursors (Gautheret \& Lambert, 2001; Legendre et al., 2005). Another study have been proposed starting with the BLAST searching with known pre-miRNA sequences followed by assessment of structure information (Wang et al., 2005). Since in many cases though the miRNA sequences may be conserved, the precursor sequences are much less conserved (Lau et al., 2001), so, searching with pre-miRNAs might lead to under estimation of the actual miRNAs present in an organism.

The present strategy uses sequence alignment of mature miRNAs, the structure conservation and assessing the position of the mature miRNAs in the pre-miRNA. Starting with the known mature miRNAs from an organism as query, homologues were searched in a related organism allowing few mismatches depending on their phylogenetic distance. The pre-miRNA and their potentiality to form stem loop structure were assessed further and finally the stem-loop secondary structure was confirmed using MFOLD (Zuker, 2003), followed by assessing the preferable position of the miRNA in the pre-miRNA secondary structure. As an application, about 91 probable miRNA sequences have been identified in Anopheles gambiae genome starting from D. melanogaster miRNAs.

\section{MATERIALS AND METHODS}

Sources of nucleotide sequences. All available $D$. melanogaster miRNAs and the pre-miRNA sequences (79 in number) were selected from the ftp site (Griffiths-Jones, 2004) (ftp://ftp.sanger.ac.uk/pub/ databases/Rfam/miRNA/).

The complete genome sequence of $A$. gambiae are arranged in 8987 (Accession No. AAAB01000001 to AAAB01008987) scaffolds for downloading at FTP site at NCBI (ftp://ftp.ncbi.nih.gov/genbank/genomes/Anopheles_Gambiae/Assembly_scaffolds/)(Holt et al., 2002). Out of these 93 large scaffolds, covering $82 \%$ of the total genome, were selected. Remaining 8894 scaffolds, covering $18 \%$ of the genome were not taken into the analysis because of their small size and large number and also to minimize the miRsearch screening time.

Strategy of miRsearch. The computational screening of miRNA was executed through the program written in Perl scripts (Fig. 1), followed by the miRNA characteristics based screening algorithm (Fig. 2), the entire algorithm being named as miRsearch. Using Drosophila miRNA as query sequence, genome of A. gambiae, which belongs to the same order diptera, was searched with a user defined score $(\mathrm{S})$. The score $\mathrm{S}$ is based on the number of mismatches and defined as:

$S=2^{*}$ [length of miRNA-2*(no. of mismatches)].

The number of mismatches in case of organisms belonging to same genus but different species, (for example, C. elegans and C. briggsae pair) was chosen to be zero, but we have relaxed it to allow for 3 mismatches for D. melanogaster and A. gambiae pair as they belong to the same order diptera.

Next, the searching and selection of pre-miRNAs were done using the following algorithm. The query sequence $(\mathrm{q}[\mathrm{i}])$ of size $\mathrm{n}$ nucleotide was placed along the column and the input sequence of same size of the query (target $t[j]$ ) was passed along the row, so as to form a $n \times n$ matrix $(M)$. For $i=j=0$ to $\mathrm{n}-1, \mathrm{q}[\mathrm{i}]$ was compared with $\mathrm{t}[\mathrm{j}]$ for perfect matching and assigned a score of +2 and otherwise -2 , the scores were placed along $M[i][j]$ for $i=j=0$ to $n-1$. If the trace of the matrix is greater than the user given score $S$, the reverse match was searched for from -80 to -1 from the first base of the target sequence, to find whether other arm of the hairpin loop precursor miRNA is in the upstream of the target sequence else from +1 to +80 from the last base of the target sequence to find whether other arm of the hairpin loop pre-miRNA is in the downstream of the target sequence. For reverse matching, the reverse complement of $\mathrm{q}[\mathrm{i}]$ was searched with a different scoring system. As the pre-miRNAs are known to form a stable hairpin loop structure, so for A-T base pairing a reward of +2 and for $\mathrm{G}-\mathrm{C}$ pairing a reward +3 
was given otherwise the reward was taken as 0 . If the reverse matching score is greater or equal to $R$, then the precursor sequence was reported with the lower and upper co-ordinates. The assigned score $\mathrm{R}$ of the reverse matching was determined by training the program to find the all known $D$. melanogaster miRNAs.

Both the forward and reverse complement sequences of the scaffolds were searched for the analysis of miRNAs. The selected sequences representing probable candidates were then examined through NCBI map viewer for their possible location (http://www.ncbi.nlm.nih.gov/mapview/map_search. cgi?taxid=7165) and those located in the exonic regions were eliminated.

The candidate pre-miRNAs were then filtered through the RNA folding program MFOLD (Zuker, 2003) (http://www.bioinfo.rpi.edu/applications/ $\mathrm{mfold} / \mathrm{old} / \mathrm{rna} /$ form3.cgi) selecting the cut-off values on the basis of known D. melanogaster pre-miRNAs (Fig. 2). The characteristics observed from the MFOLD output of D. melanogaster pre-miRNAs are: (i) the predicted mature miRNAs are within the long helical arm of the secondary structure of pre-miR$\mathrm{NA}$; (ii) $\Delta \mathrm{G} \leq-21.0 \mathrm{kcal} / \mathrm{mole}$; (iii) largest helical arm contained at least $23 \mathrm{bp}$ sequence. Considering these observations, the MFOLD output of each A. gambiae pre-miRNA was examined. A structure is accepted as probable miRNA if (a) $\Delta \mathrm{G} \leq-21.0 \mathrm{kcal} / \mathrm{mole}$, (b) the longest helical arm contains at least (20-29) bp depending on miRNA sequence length and (c) the predicted miRNAs are within the long helical arm.

The search program will be available from the authors on request.

\section{RESULTS}

Computational prediction of miRNAs by miRsearch. Observations have suggested that mature miRNA sequences are phylogenetically conserved and have characteristic stem-loop secondary structure. Based on this miRsearch used a homologous sequence searching strategy to identify the primary sequence which was simultaneously examined for the capability of formation of stem-loop secondary structure and subsequently MFOLD was used for final prediction of miRNA as described in detail in methods. Using D. melanogaster (Dme) miRNA as input, it searches homologous sequences with a maximum of 3 mismatches in the scaffolds of $A$. gambiae sequences. Homologous sequences, with 3 mismatches, may be present in many places in the genome, all of which may not have the capability of forming stem-loop precursor structure characteristics of pre-miRNAs. To eliminate those sequences, which do not form typical pre-miRNA structures, reverse complement of the homologue of miRNA sequences (reverse match) were searched at a position -80 to +80 from the matched sequence. To assign a proper score value to the reverse matching sequence, the program was trained with all Dme miRNA sequences and we empirically set the minimum score value obtained from Dme sequences as the cut off score for A. gambiae miRNA (Fig. 1). As miRNA genes can be located on either strand, we searched each sequence in both the forward strand as well as in its reverse complement. Using a total of 79 mature miRNA sequences in the miRbase sequence database and 93 scaffolds of A. gambiae, we have detected 489 sequences, which had homologous miRNA sequences and their pre-miRNAs are capable of forming of stem-loop secondary structures. This total set was viewed through Mapviewer to identify their location in the A. gambiae genome. Only 13 of these were found to present in the exonic region of genes and were excluded from the set as miRNAs are not supposed to be present in exonic region. Further evaluation of the quality of stem-loop formation of the remaining 476 pre-miRNA sequences was assessed through the RNA folding program MFOLD and

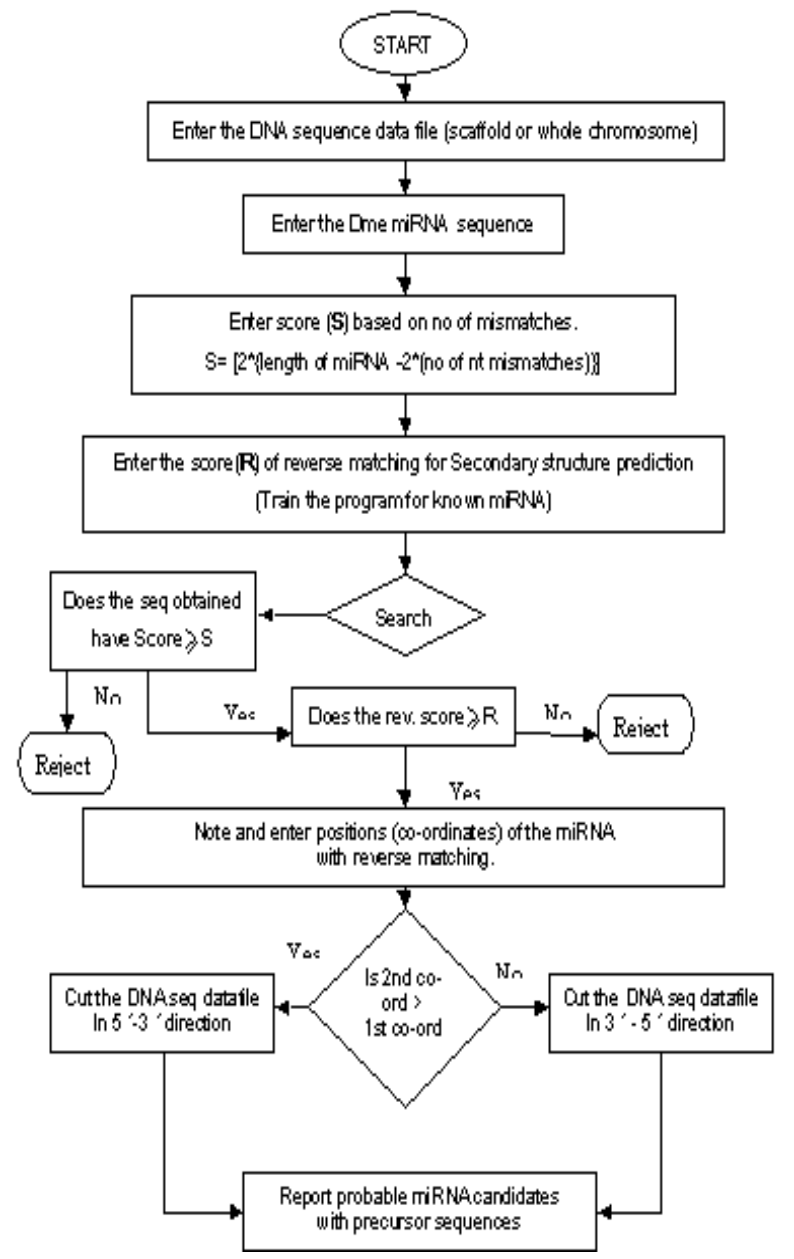

Figure 1. Flowchart for the identification of probable miRNA candidates with precursor sequences. 
Table 1. miRsearch predicted A. gambiae miRNAs $100 \%$ similar to already reported miRNA

\begin{tabular}{|c|c|c|c|c|}
\hline \multirow{2}{*}{$\begin{array}{l}\text { Sl } \\
\text { No. }\end{array}$} & \multicolumn{3}{|c|}{ Position of miRNAs in A. gambiae } & \multirow[t]{2}{*}{$100 \%$ Homologous miRNA } \\
\hline & $\begin{array}{l}\text { Chromosome } \\
\text { No. }\end{array}$ & $\begin{array}{l}\text { Scaffold } \\
\text { No. }\end{array}$ & Co-ordinate & \\
\hline 1 & $2 \mathrm{~L}$ & AAAB01008960 & $1144915(-)$ & miR-9a \\
\hline $2^{*}$ & $2 \mathrm{~L}$ & AAAB01008960 & $11605587(+)$ & $\mathrm{miR}-2 \mathrm{a} \mathrm{miR}-2 \mathrm{bmiR}-2 \mathrm{c}$ \\
\hline $3^{*}$ & $2 \mathrm{~L}$ & АAАB01008960 & $11607193(+)$ & miR-2a miR-2b miR-2c \\
\hline 4 & $3 R$ & AAAB01008964 & $6508743(+)$ & miR-9c miR-9a \\
\hline 5 & $3 R$ & AAAB01008980 & $2232282(+)$ & $\operatorname{miR}-275$ \\
\hline 6 & $3 R$ & AAAB01008944 & $3005476(-)$ & $\operatorname{miR}-133$ \\
\hline 7 & $3 R$ & AAAB01008984 & $10212876(+)$ & $\operatorname{miR}-124$ \\
\hline 8 & $3 R$ & AAAB01008964 & $6511654(+)$ & $\operatorname{miR}-79$ \\
\hline 9 & $3 R$ & AAAB01008980 & $2241140(+)$ & miR-305 \\
\hline 10 & $3 R$ & AAAB01008964 & $2129745(+)$ & $\operatorname{miR}-125$ \\
\hline $11^{*}$ & $2 \mathrm{~L}$ & AAAB01008960 & $3418059(-)$ & miR-281-2 miR-281a miR-281b \\
\hline 12 & $2 \mathrm{~L}$ & AAAB01008948 & $3223987(-)$ & $\operatorname{miR}-7$ \\
\hline 13 & $3 \mathrm{~L}$ & AAAB01008986 & $3020315(-)$ & $\mathrm{miR}-8$ \\
\hline 14 & $3 \mathrm{~L}$ & AAAB01008986 & $7425449(-)$ & miR-307 \\
\hline 15 & $3 R$ & AAAB01008984 & $6108942(+)$ & miR-14 \\
\hline 16 & $3 R$ & AAAB01008964 & $1830797(+)$ & miR-1 \\
\hline 17 & $2 \mathrm{~L}$ & AAAB01008960 & $4977122(-)$ & miR-219 \\
\hline $18^{*}$ & $2 \mathrm{~L}$ & AAAB01008960 & $4977170(+)$ & miR-276a miR-276b \\
\hline 19 & $2 \mathrm{~L}$ & АAАB01008960 & $5047343(+)$ & miR-276a miR-276b \\
\hline $20^{*}$ & $2 \mathrm{~L}$ & AAAB01008960 & $5047365(+)$ & $\mathrm{miR}-276 \mathrm{a} \mathrm{miR}-276 \mathrm{~b}$ \\
\hline $21^{*}$ & $2 \mathrm{~L}$ & AAAB01008960 & $5047385(+)$ & miR-276a \\
\hline 22 & $2 \mathrm{R}$ & АAАB01008879 & $2159581(+)$ & miR-315 \\
\hline 23 & $2 \mathrm{~L}$ & AAAB01008807 & $11606723(+)$ & miR-13b miR-13a \\
\hline $24^{*}$ & $2 \mathrm{R}$ & АААВ01008987 & $3180493(-)$ & miR-11 \\
\hline 25 & $2 \mathrm{R}$ & AAAB01008850 & $89732(-)$ & miR-10 \\
\hline 26 & $2 \mathrm{R}$ & AAAB01008898 & $1736641(-)$ & $\operatorname{miR}-279$ \\
\hline $27^{*}$ & $2 \mathrm{R}$ & AAAB01008859 & $3300849(+)$ & miR-34 \\
\hline 28 & $2 \mathrm{R}$ & AAAB01008850 & $675477(+)$ & miR-iab-4-5p \\
\hline $29^{*}$ & $2 \mathrm{R}$ & AAAB01008850 & $675511(+)$ & miR-iab-4-3p \\
\hline 30 & $2 \mathrm{R}$ & AAAB01008851 & $1388847(-)$ & $\operatorname{miR}-283$ \\
\hline 31 & $3 R$ & AAAB01008964 & $2125233(+)$ & miR-100 \\
\hline $32^{*}$ & $x$ & AAAB01008846 & $10052753(+)$ & $\operatorname{mir}-33$ \\
\hline 33 & $2 \mathrm{~L}$ & AAAB01008960 & $3418021 \quad(-)$ & mir-281 \\
\hline 34 & $2 \mathrm{~L}$ & AAAB01008960 & $3551786(+)$ & $\operatorname{mir}-282$ \\
\hline 35 & $2 \mathrm{R}$ & AAAB01007923 & $7996(-)$ & mir-92b \\
\hline 36 & $2 \mathrm{R}$ & AAAB01007923 & $22576 \quad(-)$ & $\operatorname{mir}-92 a$ \\
\hline 37 & $2 \mathrm{R}$ & AAAB01008888 & $202598 \quad(+)$ & mir-92a \\
\hline 38 & $2 \mathrm{R}$ & AAAB01008888 & $222094(+)$ & mir-92b \\
\hline 39 & $3 \mathrm{~L}$ & AAAB01008986 & $7425449 \quad(-)$ & mir-307 \\
\hline 40 & $3 \mathrm{~L}$ & AАAB01008986 & $1063490 \quad(+)$ & $\operatorname{mir}-308$ \\
\hline 41 & $3 R$ & AAAB01008964 & $2129204 \quad(+)$ & let-7 \\
\hline
\end{tabular}

some selection procedure set empirically by studying Dme miRNAs (Material and Methods, Fig. 2). A total of 91 pre-miRNA sequences have been finally identified as the probable candidate A. gambiae miRNA after passing through the entire miRsearch.

Out of 91 A. gambiae miRNAs, 41 have $100 \%$ similarity to the Dme miRNA (Table 1). One miRNA, which was $85.7 \%$ similar to Dme miRNA (dme-mir-33), was $100 \%$ similar to the hsa-mir33 miRNA (Homo sapiens). Two of the Dme miRNAs viz. miR-9a and miR-2a were conserved both in sequence and in their location in chromosome 2L. Other predicted miRNAs were not conserved in their chromosomal location (not shown). The miRNA gene cluster of miR-276a found in chromosome $2 \mathrm{~L}$ of $D$. melanogaster was detected in chromosome $3 \mathrm{~L}$ in A. gambiae. The locations of the predicted miRNA were also recorded (Table $1,2)$. Remaining 50 miRNAs are probable newly identified A. gambiae miRNA having potential for the formation of hairpin secondary structure with high degree of MFE $(\Delta G)$ and more than $85 \%$ sim- 
Table 2. Probable newly identified A. gambiae miRNAs predicted by miRsearch

\begin{tabular}{|c|c|c|c|}
\hline $\begin{array}{l}\text { Sl } \\
\text { No. }\end{array}$ & Probable miRNA (Anopheles gambiae) & Position (chr no., scaffold \& co-ordinates) & $\begin{array}{l}\text { Closest homolo- } \\
\text { gous miRNA }\end{array}$ \\
\hline 1 & TCACTGGGCAAAGTTTGTCGCA & 2L AAAB01008968 494152 & miR-3 \\
\hline 2 & ATCACAGCCAGCTTTGAAGAGC & 2L AAAB01008960 11606893 & $\operatorname{miR}-2 \mathrm{c}$ \\
\hline 3 & GATCACATGCAGCTTTGAGGAGA & 2L AAAB01008960 7464065 & miR-2b \\
\hline 4 & TATCACAGCCAGCTTTGAAGAGC & 2L AAAB01008960 11606893 & miR-2 \\
\hline 5 & TCAGGCATCTGCAGTAGCGCACG & 2L AAAB01008960 3748081 & $\operatorname{miR}-275 b$ \\
\hline 6 & CAGCGAGGTATAGAGTTCCTATG & 2LAAAB01008960 4977122 & $\operatorname{miR}-276$ \\
\hline 7 & TGTCATGGAATTGCTCTCTTTAT & 2L AAAB01008960 3418021 & miR-281 \\
\hline 8 & AATCTAGCCTCTTCTAGGCTTTGTCTGT & 2L AAAB01008960 3551786 & $\operatorname{miR}-286$ \\
\hline 9 & CAGCGAGGTATAGAGTTCCTATG & 2L AAAB01008960 4977122 & $\operatorname{miR}-276$ \\
\hline 10 & TGTGTTGAAAATCATGTGCAA & 2L AAAB010089609175668 & miR-287 \\
\hline 11 & TGTGTTGAAAATCATTTGTAA & 2L AAAB01008960 3952821 & miR-287 \\
\hline 12 & TGAGACAATTTTGAAAGCTGAGT & 2L AAAB01008807 4023317 & miR-bantam \\
\hline 13 & TATCACAGCCAGCTTTGAAGAGC & 2L AAAB01008807 11606893 & miR-2 \\
\hline 14 & CCTTATTATGCTTTCGCCCCG & 2R AAAB01008844 938949 & miR-184 \\
\hline 15 & ATTGCACTTGTCCCGGCCTGC & 2R AAAB01007923 7996 & miR-92b \\
\hline 16 & ATATTGCACTTGTCCCGGCCTAT & 2R AAAB01007923 22576 & miR-92a \\
\hline 17 & TATTGCACTTGTCCCGGCCTAT & 2R AAAB01008888 202598 & miR-92a \\
\hline 18 & TGGCAGTCCGGTTTGCTGGTTG & 2R AAAB01008987 4283494 & miR-34 \\
\hline 19 & TCGCTCCATTCGCAATCAGTGC & 2R AAAB01008859 1718173 & $\operatorname{miR}-285$ \\
\hline 20 & AATTGCACTTGTCCCGGCCTGC & 2R AAAB01007923 7996 & miR-92b \\
\hline 21 & AATTGCACTTGTCCCGGCCTGC & 2R AAAB01008888 222094 & miR-92b \\
\hline 22 & AAACGGACGAAAGTCCCACCGA & 3L AAAB01008986 7850785 & miR-212 \\
\hline 23 & TGTGTTGAAAATCATGTGCAC & 3L AAAB01008816 932527 & miR-287 \\
\hline 24 & CGTGTTGAAAATCGTGTGCAA & 3L AAAB01008823 2635002 & $\operatorname{miR}-287$ \\
\hline 25 & TGTGTTGAAAATCATTTGAAA & 3L AAAB01008823 3200243 & miR-287 \\
\hline 26 & TAGCACCATTCGAAATCAGTAC & 3L AAAB01008986 2720706 & miR-285 \\
\hline 27 & AGAGATCATTTTGCAAGATGATT & 3L AAAB01008816 577447 & miR-bantam \\
\hline 28 & TTTGTTGAAAATCCTTTGCAA & 3L AAAB01008986 7693438 & $\operatorname{miR}-287$ \\
\hline 29 & TGTGTTGAAAATCATGTGGAC & 3L AAAB01008986 8813783 & miR-287 \\
\hline 30 & TTATCTCAATTGGTTAGTGTGAG & 3L AAAB01008966 2521934 & miR-304 \\
\hline 31 & AATCACAGGAGTATACTGTGAGA & 3L AAAB01008986 1063490 & miR-308 \\
\hline 32 & ACAGTTTTTTTCCСТCTCCTA & 3R AAAB01008980 11682415 & miR-14 \\
\hline 33 & TCAGTCTTTTACTCTCCACTA & 3R AAAB01008980 14365242 & $\operatorname{miR}-14$ \\
\hline 34 & AACCCGTAGATCCGAACTTGT & 3R AAAB01008964 2125233 & miR-100 \\
\hline 35 & GCTTTGGTAATCTAGCTTTATGA & 3R AAAB01008964 6511651 & $\operatorname{miR}-9$ \\
\hline 36 & TCACTGGGCAAAGTTTGTCGCA & 3R AAAB01008980 6863595 & $\operatorname{miR}-3$ \\
\hline 37 & CATCACAGCCCAATTTGATGAGC & 3R AAAB01008980 5038400 & miR-2a \\
\hline 38 & CCTTATCATTCTTTCGCCCCG & 3R AAAB01008980 6487250 & $\operatorname{miR}-184$ \\
\hline 39 & TGGACGGAGAACTGATAAGGG & 3R AAAB01008980 64872876 & miR-184 \\
\hline 40 & TCGGTGGGACTTTGGTGTGTTT & 3R AAAB01008984 6174056 & miR-278 \\
\hline 41 & AGTTTTTATGTTATATATGATATGATA & 3R AAAB01008839 416964 & miR-280 \\
\hline 42 & TGACTAGACCGAACACTCGCGTC & 3R AAAB01008980 6864288 & miR-286 \\
\hline 43 & TGTGTTGAAAATCATGTGCAA & 3R AAAB01008944 2423617 & $\operatorname{miR}-287$ \\
\hline 44 & TGTGTTGAAAATCATGTGCAA & 3R AAAB01008980 10846168 & miR-287 \\
\hline 45 & TGAGGTAGTTGGTTGTATAGT & 3R AAAB01008964 2129204 & miR-let7 \\
\hline 46 & ТСТСТСТТТСТСТСТСТССТА & X АААВ01008847 1241766 & miR-14 \\
\hline 47 & TGTGTTGAAAATCATGTGCAA & X АAАB01008846 4269543 & miR-287 \\
\hline 48 & GTGAGCAAATATTCAGGTGTG & X АAАB01008846 11047618 & miR-87 \\
\hline 49 & TGGCAAGATGTTGGCATAGCTA & X АААВ01008847 3057089 & miR-72 \\
\hline 50 & TGGCAAGATGTTGGCATAGCTAA & X AAAB01008847 357089 & miR-72 \\
\hline
\end{tabular}

ilar with the already predicted miRNAs (Table 2). In some cases, same predicted miRNA was obtained using different Dme miRNAs as the query sequence (Table 1 and 2).
Interestingly, although the miRNA sequences of Drosophila and Anopheles were 100\% similar (Table $1)$, the sequences and structures of corresponding pre-miRNAs from Drosophila and Anopheles were not conserved to that extent (Fig. 3). This holds good for 


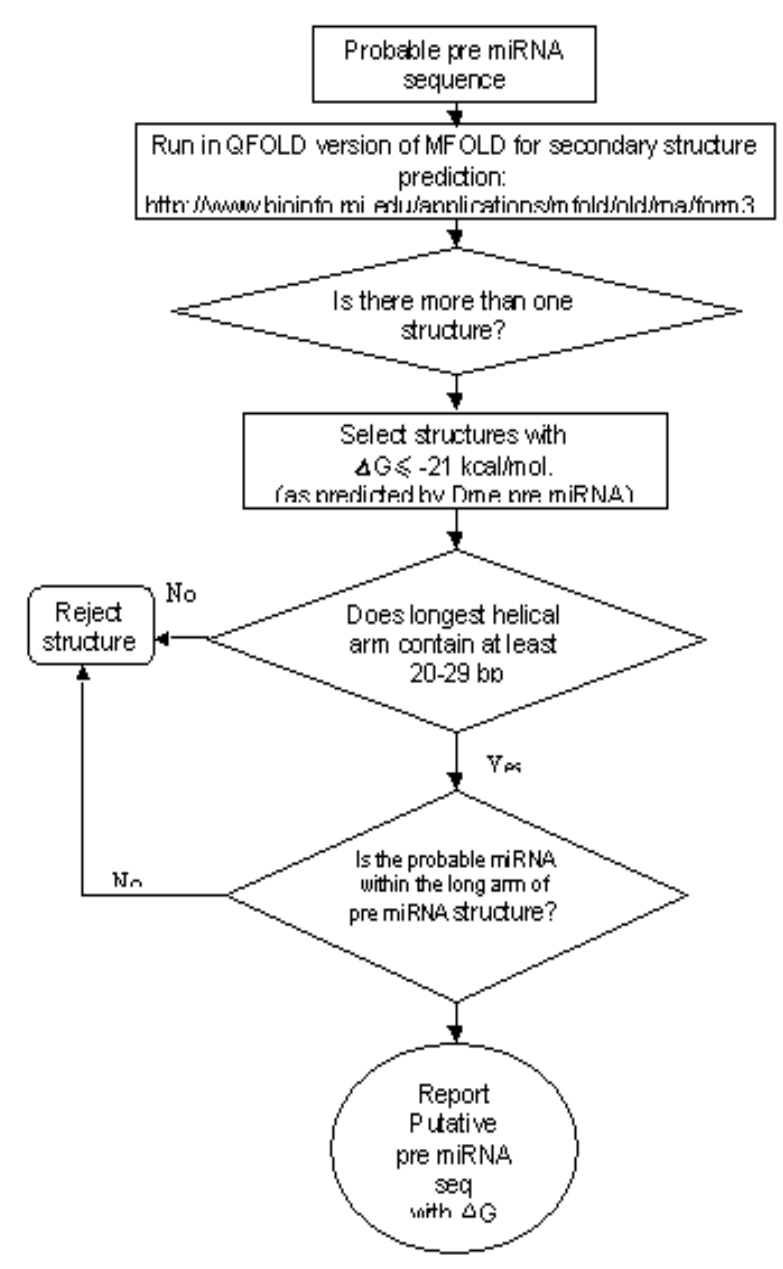

Figure 2. Flowchart for prediction of putative miRNAs based on the miRNA characteristics.

each of the 30 miRNA sequences. This analysis supports our strategy for the miRNA detection, which is based on homology search with the mature miRNA sequences rather than precursor miRNA sequences.
Out of the total 83 A. gambiae miRNA reported so far (Griffiths-Jones et al., 2004), we have detected 31 miRNAs. A set of 11 Anopheles miRNAs were found to be $100 \%$ similar to Dme miRNAs, was missed in our study, which is due to exclusion of scaffolds covering $18 \%$ of the genome in our study. Moreover, we have identified 10 new miRNAs having $100 \%$ similarity with the other species miRNAs. This data suggest that, use of only Dme miRNAs as query data set would have detected at least 42 of the predicted miRNAs, new 10 miRNAs and 50 putative novel miRNAs. However, use of all the known miRNA sequences, proper choice of mismatches for each species and considering the whole genome sequences will enhance the efficiency of miRsearch for the identification of new miRNAs.

\section{DISCUSSION}

Informatics approach used so far to identify miRNA involves alignment of genomes of two closely related species to find conserved regions followed by identification of stem-loop precursor transcripts capable of processing and forming about 22 nt mature RNA (Lai et al., 2003; Lim et al., 2003a). In our approach we have eliminated the whole genome alignment step and instead have used the following steps: (i) searching for homologues of known mature miRNA in one organism (Dme) to the genome of another related organism $(A g a)$, after allowing some mismatches, depending on phylogenetic distance between them, (ii) assessing the capability to form stem-loop precursors or structures and finally (iii) observing the preferable position of the mature miRNA in the secondary structure of pre-miRNA. Such an approach is most useful when the complete set of miRNA in one organism is available along
Ano mir-13b:

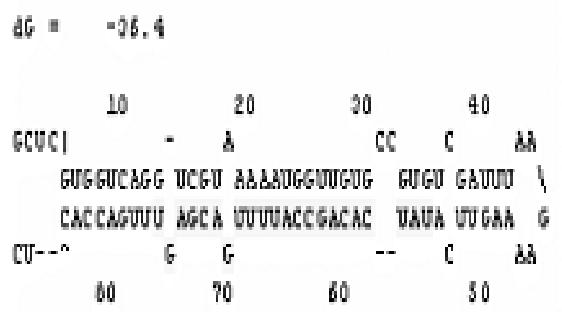

Ano mir-100:

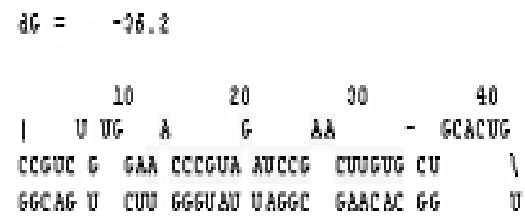

Dme mir-13b:

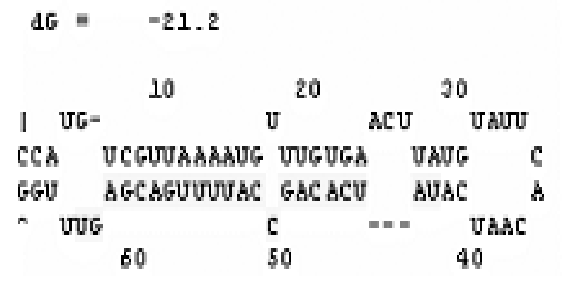

Hsa mir-100

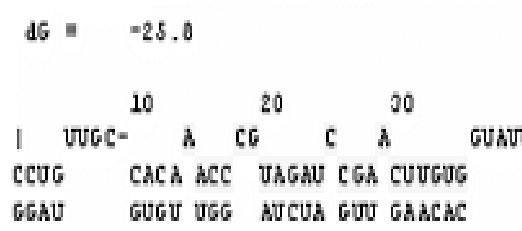

Figure 3. MFOLD generated secondary structure of pre-miRNA corresponding to D. melanogaster miRNA (Dme mir-13b) and H. sapiens miRNA (Hsa mir-100) and their corresponding $100 \%$ si$\checkmark$ milar A. gambiae miRNA preA dicted by miRsearch. 
with the genome sequence of a related organism. The chances of getting false positive are little. While comparing with the other methods for the detection of A. gambiae miRNAs, miRAlign (Wang et al., 2005) used the complete set of miRNAs available in the database and the whole genome sequence of $A$. gambiae, which is available at NCBI through BLAST search and MapViewer, whereas we have used only the Dme miRNAs and $82 \%$ of the genome of A. gambiae. MiRAlign detected 59 putative miRNAs, out of these $37(44.6 \%)$ are already predicted (GriffithsJones, 2004). Our study have detected 91 total miRNAs, out of these 41 are already predicted. For comparison if we study the whole genome of A. gambiae, we would have detected at least $52(63 \%)$ already predicted miRNAs. As we have suggested earlier, the search for homologues by BLAST starting with pre-miRNAs as query may miss many of the miRNAs as the pre-miRNA sequences of $100 \%$ similar mature miRNAs differ considerably (Fig. 3).

However, in this approach we may miss some of the miRNAs, which are exceptionally divergent and may not be homologous at all to the available miRNAs. The above program may be accommodated to identify miRNAs in not so related organism also (as many of the miRNAs are evolutionarily conserved) by increasing the number of mismatches in miRsearch, although the chances of getting a large number of false positives will be high. To reduce this, further filtering techniques need to be devised, which is currently under investigation.

\section{Acknowledgement}

R.C. is indebted to Council of Scientific and Industrial Research (C.S.I.R) for Research fellowship.

\section{REFERENCES}

Altschul SF, Gish W, Miller W, Myers EW, Lipman DJ (1990) Basic local alignment search tool. J Mol Biol 215: 403-410.

Bartel B, Bartel DP (2003) MicroRNAs: at the root of plant development? Plant Physiol 132: 709-717.

Bartel DP (2004) MicroRNAs: genomics biogenesis mechanism and function. Cell 116: 281-297.

Bonnet E, Wuyts J, Rouze P, Van de Peer Y (2004) Detection of 91 potential conserved plant microRNAs in Arabidopsis thaliana and Oryza sativa identifies important target genes. Proc Natl Acad Sci USA 101: 11511-11516.

Gautheret D, Lambert A (2001) Direct RNA motif definition and identification from multiple sequence alignments using secondary structure profiles. J Mol Biol 313: 1003-1011.

Griffiths-Jones S (2004) The microRNA registry. Nucleic Acids Res 32: D109-D111.

Holt RA, Subramanian GM, Halpern A, Sutton GG, Charlab R, Nusskern DR et al. (2002) The genome sequence of the malaria mosquito Anopheles gambiae. Science 298: 129-149.

Jones-Rhoades MW, Bartel DP (2004) Computational identification of plant microRNAs and their targets including a stress-induced miRNA. Mol Cell 14: 787-799.

Lagos-Quintana M, Rauhut R, Lendeckel W, Tuschl T (2001) Identification of novel genes coding for small expressed RNAs. Science 294: 853-858.

Lai EC, Tomancak P, Williams RW, Rubin GM (2003) Computational identification of Drosophila microRNA genes. Genome Biol 4: R42.

Lau NC, Lim LP, Weinstein EG, Bartel DP (2001) An abundant class of tiny RNAs with probable regulatory roles in Caenorhabditis elegans. Science 294: 858-862.

Lee RC, Ambros V (2001) An extensive class of small RNAs in Caenorhabditis elegans. Science 294: 862-864.

Lee RC, Feinbaum RL, Ambros V (1993) The C. elegans heterochronic gene lin-4 encodes small RNAs with antisense complementarity to lin-14. Cell 75 843-854.

Legendre M, Lambert A, Gautheret D (2005) Profile-based detection of microRNA precursors in animal genomes. Bioinformatics 21: 841-845.

Lewis BP, Shih IH, Jones-Rhoades MW, Bartel DP, Burge CB (2003) Prediction of mammalian microRNA targets. Cell 115: 787-798.

Lim LP, Glasner ME, Yekta S, Burge CB, Bartel DP (2003a) Vertebrate microRNA genes. Science 299: 1540.

Lim LP, Lau NC, Weinstein EG, Abdelhakim A, Yekta S, Rhoades MW et al. (2003b) The microRNAs of Caenorhabditis elegans. Genes Dev 17: 991-1008.

Moss EG, Lee RC, Ambros V (1997) The cold shock domain protein LIN-28 controls developmental timing in C. elegans and is regulated by the lin-4 RNA. Cell 88 : 637-646.

Moss EG, Poethig RS (2002) MicroRNAs: something new under the sun. Curr Biol 12: R688-R690.

Ohler U, Yekta S, Lim LP, Bartel DP, Burge CB (2004) Patterns of flanking sequence conservation and a characteristic upstream motif for microRNA gene identification. RNA 10: 1309-1322.

Pasquinelli AE, Reinhart BJ, Slack F, Martindale MQ, Kuroda MI, Maller B et al. (2000) Conservation of the sequence and temporal expression of let-7 heterochronic regulatory RNA. Nature 408: 86-89.

Reinhart BJ, Slack FJ, Basson M, Pasquinelli AE, Bettinger JC, Rougvie AE et al. (2000) The 21-nucleotide let-7 RNA regulates developmental timing in Caenorhabditis elegans. Nature 403: 901-906.

Slack FJ, Basson M, Liu Z, Ambros V, Horvitz HR, Ruvkun G (2000) The lin-41 RBCC gene acts in the C. elegans heterochronic pathway between the let-7 regulatory RNA and the LIN-29 transcription factor. Mol Cell 5: 659-669.

Wang X, Zhang J, Li F, Gu J, He T, Zhang X et al (2005) MicroRNA identification based on sequence and structure alignment. Bioinformatics 21: 3610-3614.

Wang XJ, Reyes JL, Chua NH, Gaasterland T (2004) Prediction and identification of Arabidopsis thaliana microRNAs and their mRNA targets. Genome Biol 5: R65.

Weber MJ (2005) New human and mouse microRNA genes found by homology search. FEBS J 272: 59-73.

Zuker M (2003) Mfold web server for nucleic acid folding and hybridization prediction. Nucleic Acids Res 31: 3406-3415. 